\title{
Guest editorial: Digital solutions to bridging the gap between health services and workforce in rural areas
}

\section{Introduction to this special issue}

The papers in this special issue of Health Education explore the changing role of digital technology in rural health. Submissions were invited that provided evidence of digital interventions aimed at increasing access to education, training and development or support for rural health professionals. In particular, articles examine enablers and barriers to using digital technology for this purpose and aim to better understand the conditions under which digital support to rural locations can work and make recommendations about appropriate models.

Recruitment and retention of health professionals to rural and remote areas is a challenge in Australia and globally. A strong rural health workforce, particularly supporting public health and primary prevention, is a vital component of improving rural communities and rural population health (reference). And yet, high staff turnover and long-term unfilled positions limit the health services that can be provided (Onnis, 2019; Wakerman et al., 2019). There is without doubt a significant amount of scholarship that has, over time, sought to examine the attraction, recruitment and retention of health professionals to rural locations (Ogden et al., 2020; Onnis, 2016; Russell et al., 2017).

The literature indicates the factors and solutions are complex (Malatzky et al., 2019; Russell et al., 2017). In recent research with rural and remote allied health professionals, the most cited factors that negatively influence retention were lack of professional development opportunities, professional isolation and insufficient supervision (Campbell et al., 2012; Cosgrave, 2020). For rural and remote clinicians, quality education is often difficult to access because of geographic isolation, travel, time, expense constraints and lack of an onsite educator (Calleja et al., 2022).

There is also an emerging literature that relates to the acceptance and advancement of telehealth and digital technologies to positively impact on professional practices (Pit et al., Ramsden et al., 2021, Safi et al., 2018). However, to date there is limited examination of the impact of digital access on professional education and support to health professionals in rural areas. Digital access is a complex and multi-dimensional concept, as it includes access to hardware, software and connectivity to the Internet as well as meaningful, high-quality and culturally relevant content and the ability to create, share and exchange knowledge (Willems et al., 2019).

This special issue is the result of a wonderful group of people's time and considerable expertise that enabled this publication. Authors of the paper thank the authors for interesting insights into the impact of technology on rural health, the reviewers for valuable feedback on all the manuscripts, and attention to detail, guidance and experience from the great team of Health Education staff and copy and lead editors is greatly appreciated. The authors thank the reviewers, authors, the team of Health Education staff and copy and lead editors for encouragement and support as the authors navigated the processes and systems to bring together this special issue.

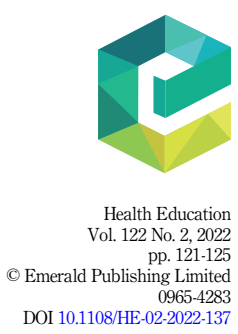


$\mathrm{HE}$

122,2

It is from this lens we explore health education in the digital age, covering current concerns and providing evidence in relation to initiatives and ideas in line with Health Education's focus on healthier lives. Focussing on the impact of digital technology on the factors and initiatives that facilitate access to education training and support in rural areas, we aim to use our research to influence real world change in people's health and well-being in rural areas/that contribute to the reduction of health inequalities. It is important to stay at the forefront of this scholarship and to continue to explore and understand where the challenges and opportunities lie.

\section{Overview of the papers}

There are seven papers in this special issue. The papers utilise different approaches to critical scholarship and provide insights into digital technology use in India, Turkey and Australia. They demonstrate the currency and rapid adoption of the use of technology in rural health. They largely focus on asynchronous learning to take advantage of the various forms of digital and online learning in which participants learn on their own at their own pace from instruction that is not delivered in person or in real time. Asynchronous learning may include pre-recorded video lessons and webinars, readings, podcasts animations or game-based learning tasks. The seven papers are important in identifying a range of innovative online practices to support diverse areas of rural health including continuing professional education, tertiary health student training and professional support for treating paediatric autism and cancer. Several papers identify how the COVID-19 pandemic has fast-tracked developments in areas including mobile phone innovations in rural India, the pragmatic development of multi-disciplinary tertiary programs in Australia and nurse and midwifery training in Turkey.

The first paper by Ramsden, Colbran, Christopher and Edwards is a scoping review of the peer-reviewed literature to determine, "The role of digital strategies in providing education, training, continuing professional development and support to the rural health workforce". The authors note there is mounting evidence demonstrating the potential for online platforms to address the challenges of rural health professional practice and the tyranny of distance. The focus of their investigation was on the use of online education, training and continuing professional development that is outside the undergraduate and postgraduate learning experiences of a university. The authors concluded there is value in technology for asynchronous learning in rural health, as it affords the opportunity to engage in learning at a time and location that is convenient and enables the learner to balance professional development with personal and work commitments. However, the authors also identified that this form of learning is not without its challenges in rural and remote areas, including poor Internet connection, skill level and need for technology support. Further, in most of these studies there was also a link between e-learning literacy and engagement in online learning. Therefore, it could be concluded that whilst the expansion of available e-learning resources and continuing advances in technology will enable many rural health professionals to access learning, it is of utmost importance that the intended audience is adequately supported to develop digital literacy and to put the learning into practice.

The papers in this issue take up some of the challenges with digital learning outlined in the scoping review. The article by Johnsson, Lincoln, Bundy, Costley and Bulkeley titled "Evaluation of a webinar and video-conferencing support program designed to upskill staff working with children with autism in remote areas", evaluated a novel webinar training and individual online support programme for 36 allied health, education and community support staff. The increase in "knowledge and skills" and "confidence" in working with children with autism reported by participants was positively related to the number of webinars the participants accessed (asynchronous learning). Synchronous learning via individual online 
sessions was accessed by a small number of participants, mostly allied health professionals. The authors concluded that technology may provide a low-cost and widely accessible platform for providing education and support for staff in these areas. This pilot study progressed the scholarship around how asynchronous learning can be effective in addressing inequity by increasing programme access and flexibility for remote rural health professionals. However, it is important to note that some barriers to engaging in the programme were identified including workload and scheduling.

Two papers refer to the growth and acceleration of the use of digital technology/ innovation and its impact in rural settings during COVID-19 (Cox et al., and Venkataraghavan et al.). The study by Cox et al., titled "Co-designing multi-disciplinary telehealth education for on-line learning, focusses on students in the tertiary education sector". The authors note the role of telehealth as an adjunct to face-to-face health service delivery in providing improved access to healthcare for residents in more rural and remote settings. They identified that students graduating from health courses and current health professionals need to have some proficiency in using telehealth. This need was accelerated with COVID-19 as health care professionals were left with no option but to transform their service into a virtual clinic despite a lack of knowledge and experience in this mode of delivery. However, there is limited accessibility to multi-disciplinary-based material to meet this need, and as a consequence, the authors experienced an increased number of clinicians and students seeking out online courses in telehealth to inform and upskill them during this time. The authors present a case study that demonstrates the value of a co-design process and key learning design choices in online course development to meet the educational needs of learners from broad disciplinary backgrounds, in various stages of learning/understanding of telehealth and/or requiring a practice-based resource in the context of a rapidly changing policy environment. Initial results from the study highlight the potential of an online selfpaced course such as the one described to contribute to "introductory" competency development in telehealth.

The paper by Venkataraghavan et al., titled “'Through their eyes, I can work'- Rural physicians' perceptions about mobile phone use among community health workers (CHWs) a qualitative analysis" relates to the Udupi district of Karnataka, India. It examines the benefits and challenges of mobile phone use by $\mathrm{CHW}$ from the perspectives of rural medical officers' (rural physicians'). Scarcity of health professionals in rural areas, distribution of Public Health Centres and a dearth of paramedical personnel continue to be challenging in many districts of the country. Hence, it is the CHWs in the Udupi district that help bridge the gap between thousands of India's rural remote populace and the health systems. Mobile phone technology assisted the CHWs in sending real-time COVID-19 pandemic reports and data to the health systems as well as for showing patients their test results or guidelines issued by health systems and government. It is evident from the study that mobile phone technology has the capability to be an effective support tool for the CHWs. However, the article provides a critical assessment on the inequity of technology access and the challenges in using technology as a tool for supporting health and well-being. Challenges including poor network coverage, technical illiteracy and insufficient training need to be addressed. The authors offer suggestions including increasing the number of mobile towers in rural areas, providing dual SIM cards from two different service providers and providing smartphones for using voice messaging and the photo and video functions. Whilst initial results from the study highlight the potential of this approach, the authors argue for more rigorous research to identify the perspectives of all the stakeholders involved in the primary health system including the beneficiaries.

Two articles specifically appraise the role of technologies to address the challenges associated with COVID-19 (Barraclough and Pit; Uçuk and Yildirim). Barraclough and Pit provide an Australian case study outlining a multi-disciplinary teaching programme that 
$\mathrm{HE}$

122,2

was adapted to allow students from various disciplines and universities to learn together during the COVID-19 pandemic. They note that the COVID-19 pandemic led to "forced innovation" in the health education industry due to the fact that academics and students were less able to be physically present. This is an outcome of border restrictions, other movement restrictions, placement cancellations in aged care facilities and rural schools, school closures and family commitments such as children not being able to go to school or day care. Again, the value of asynchronous learning is highlighted so that students have a choice of learning online, independent of time or location. The originality of this programme centres around students from multiple universities and disciplines and various year levels learning together in a rural area over an extended period of time. Collaboration amongst universities assisted educators in rural areas to achieve a critical mass of students to enable viable teaching programs. Whilst the authors note the need for further evaluation, they conclude that a rural multi-disciplinary online education programme was found to assist in reducing some of the perceived barriers to uptake of rural practice, including lack of knowledge around rural training, limited research opportunities, fear of the unknown and a limited rural training pipeline. The authors propose guidelines for remote multi-disciplinary learning amongst health professional students, including those in medical, nursing, pharmacy, dentistry and allied health disciplines.

Uçuk and Yildirim, in their paper, "The effect of COVID-19 prevention methods training given through distance learning on state anxiety level: the case of private sector", argue that COVID-19 prevention methods training given by distance learning techniques can positively impact on the anxiety level of the workers of a company serving in the communication sector in Turkey. The authors concluded that it is important to provide training and support to those with high anxiety levels as an outcome of COVID-19 and that informative support delivered by nurses/midwives can make it easier to control anxiety. Nurses and midwives were involved in delivering supportive training activities. The authors recognise some limitations in terms of generalisability such as the relationship between the knowledge score and anxiety could not be evaluated.

In the paper, "Digital Solutions for Paediatric Oncology Health Professionals in Rural and Low-Resource Settings" Martiniuk, Challinor, Arora, Handayani, Sri and Lam note that digital solutions have a valuable role in increasing health professional knowledge, skills and empowerment. They focus on the area of diagnosing, treating and caring for children and adolescents with cancer. In relation to paediatric oncology, the authors identify that a significantly lower percentage of children will survive cancer in lowresource settings. This review sought to identify digital resources that support the training and development of the paediatric oncology workforce in resource-poor settings. This paper presents a narrative descriptive review of peer-reviewed publications and digital platforms that contribute to health professionals' education and training regarding paediatric oncology, particularly in rural and other low-resource settings. A total of 33 resources are discussed in depth and a quality assessment of the digital resources is provided. The authors anticipate that this summary of digital resources for the global paediatric oncology professional community will inform digital health investments and the design of digital innovations to meet emerging needs contributing to health workforce improvements.

\section{Conclusion}

The seven papers demonstrate important international innovations in the use of digital health, suggesting it is undoubtedly here to stay. As telehealth and other digital health technologies gain momentum, it will almost certainly have more prominent inclusion in health curricula, clinical placements and ongoing professional learning and support. This is 
particularly important in the rural context. There is room for other countries to utilise or extend the innovations showcased in these papers. For example, online support for autism and paediatric cancer can be extended to other health conditions.

The opportunity that this body of work identifies should be carefully evaluated and built upon. Although multiple barriers are outlined, technology has shown itself to be an asset for rural health professionals' in circumstances where access to ongoing rural education, training and support is not currently available to many. The complementary use of telehealth or digital platforms for professional development delivery could play a large part in retaining health professionals rurally and improving health services and outcomes if appropriate models and adequate funding are brought together.

\section{Robyn Ramsden \\ NSW Rural Doctors Network, Hamilton, Australia and School of Health and Social Development, Deakin University, Melbourne, Australia, and Michelle Lincoln Faculty of Health, University of Canberra, Canberra, Australia}

\section{References}

Calleja, P., Wilkes, S., Spencer, M. and Woodbridge, S. (2022), "Telehealth use in rural and remote health practitioner education: an integrative review”, Rural and Remote Health, Vol. 22, doi: 10. 22605/RRH6467.

Campbell, N., McAllister, L. and Eley, D. (2012), "The influence of motivation in recruitment and retention of rural and remote allied health professionals: a literature review", Rural Remote Health, Vol. 12, p. 1900.

Cosgrave, C. (2020), "The whole-of-person retention improvement framework: a guide for addressing health workforce challenges in the rural context", International Journal of Environmental Research and Public Health, Vol. 17 No. 8, p. 2698.

Malatzky, C., Cosgrave, C. and Gillespie, J. (2019), "The utility of conceptualisations of place and belonging in workforce retention: a proposal for future rural health research", Health and Place, Vol. 62, doi: 10.1016/j.healthplace.2019.102279.

Ogden, J., Preston, S., Partanen, R.L., Ostini, R. and Coxeter, P. (2020), "Recruiting and retaining general practitioners in rural practice: systematic review and meta-analysis of rural pipeline effects", Medical Journal of Australia, Vol. 213 No. 5, pp. 228-236.

Onnis, L.A. (2019), "Human resource management policy choices, management practices and health workforce sustainability: remote Australian perspectives Asia Pac", Journal of Human Resources, Vol. 57, pp. 3-23.

Russell, D.J., McGrail, M.R. and Humphreys, J.S. (2017), "Determinants of rural Australian primary health care worker retention: a synthesis of key evidence and implications for policymaking", Australian Journal of Rural Health, Vol. 25 No. 1, pp. 5-14.

Safi, S., Thiessen, T. and Schmailzl, K.J. (2018), “Acceptance and resistance of new digital technologies in medicine: qualitative study”, JMIR Research Protocols, Vol. 7 No. 12, e11072.

Wakerman, J., Humphreys, J., Russell, D., Guthridge, S., Bourke, L., Dunbar, T., Zhao, Y., Ramjan, M., Murakami-Gold, L. and Jones, M.P. (2019), "Remote health workforce turnover and retention: what are the policy and practice priorities?", Human Resources for Health, Vol. 17, p. 99.

Willems, J., Farley, H. and Campbell, C. (2019), "The increasing significance of digital equity in higher education: an introduction to the Digital Equity Special Issue", Australasian Journal of Educational Technology, Vol. 35 No. 6, pp. 1-8. 